\title{
Impairments in deductive reasoning and working memory in Parkinson's disease
}

\author{
Shelley Channon \\ Subdepartment of Clinical Health Psychology, University College (London), \\ London, UK \\ Correspondence to: S. Channon, Subdepartment of Clinical Health Psychology, \\ University College (London), Gower Street, London WC1E 6BT, UK
}

\begin{abstract}
This study was designed to investigate the nature and extent of executive impairments in medicated subjects with Parkinson's disease (PD) and matched control subjects. They performed two tasks involving strategic processing, deductive reasoning and memory updating. PD patients differed significantly from controls in solving two- and four-dimensional deductive reasoning problems, and they also showed impairment in memory updating. The findings are discussed in relation to previous studies which have shown deficits in strategic processing in PD.
\end{abstract}

Keywords: Parkinson's disease - Neurologic assessment - Problem-solving - Memory

\section{INTRODUCTION}

The investigation of executive deficits resulting from PD is of considerable interest in view of the close anatomical links between the basal ganglia and the frontal lobes (see, for example, Alexander et al., 1986). A growing body of evidence has suggested that, under certain conditions, PD subjects are impaired on executive tasks found to be sensitive to frontal lobe lesions, although PD impairments have generally been found to be less marked than those displayed by subjects with lesions to the frontal lobes, and may also differ qualitatively. Factors such as duration of the disease and use of medication may influence both the nature and severity of cognitive deficits shown (see, for example, Owen et al., 1992).

Interest has centred around characterizing the nature of executive deficits in PD. Evidence from a range of tasks led to the hypothesis (see, for example, Cools et al., 1984) that PD subjects are impaired in shifting set on tasks such as the Wisconsin Card Sorting Test (WCST) (see, for example, Bowen et al., 1975; Lees and Smith, 1983; Pillon et al., 1986; Taylor et al., 1986; Gotham et al., 1988) which is known to be sensitive to frontal lobe dysfunction (Milner, 1964). Impairment has also been reported in PD patients on a wide range of other executive tasks which involve set shifting (see, for example, Cools et al., 1984; Flowers and Robertson, 1985; Brown and Marsden, 1988; Downs et al., 1989; Owen et al., 1992; Channon et al.,
1993; Cronin-Golomb et al., 1994).

Whilst it is clear that PD patients often show impairment on tasks which involve set shifting, a range of faulty cognitive mechanisms have the potential to contribute to task impairment, and shifting set per se may not be a crucial component. The classical frontal-type error is characterized as perseveration which, most commonly, refers to the development of a cognitive set and subsequent difficulties in disengaging from this set, and shifting to a more adaptive response in an altered stimulus environment. This was described as 'stuck-in-set perseveration' by Sanderson and Albert, 1987. It seems improbable that perseveration is solely responsible for task impairment in $\mathrm{PD}$, since errors other than perseveration have been reported in PD subjects, both on the WCST and other tasks. Nevertheless, a recent study by Cronin-Golomb et al. (1994) returned to the notion of perseveration and set shifting as the fundamental impairment in PD. CroninGolomb et al. (1994) gave PD subjects several tests of problem solving, concept formation and abstract comprehension. They reported that the PD group performed normally on tests of problem solving, concept formation and abstract comprehension, with the exception of one (Poisoned Foods), which involved solving a series of deductive reasoning problems of a similar format. PD patients showed greater numbers of intrusion errors than controls on this task, but did not 
differ in rates of other errors. The authors suggested that PD patients do not show impairment in capacity for deductive reasoning per se, but that they are impaired in suppressing learned responses that are inappropriate in a changed stimulus environment, showing both 'stuck-in-set' perseveration and 'recurrent' perseveration, or errors of intrusion.

Several alternative accounts of PD impairment on executive tasks which involve set shifting have been proposed. Brown and Marsden (1988) suggested that PD is associated with impairment only when external cues for shifting set are not present, and they interpreted this as evidence of reduced resources in the Supervisory Attentional System (SAS; Norman and Shallice, 1986). This is functionally similar to the central executive (CE) component of working memory (Baddeley and Hitch, 1974), which has been linked to the prefrontal cortex. It has also been postulated that PD patients have difficulty in maintaining a stable response set against competing alternatives (Flowers and Robertson, 1985; Downes et al., 1989). Downes et al. (1989) argued in relation to their findings that PD deficits did not reflect impaired set shifting per se, but speculated that they had greater difficulty than normal in attending to previously unreinforced stimuli. The debate about the nature of PD deficits is fuelled in part by the varying nature of the tasks employed to study them, since not all tasks will be sensitive to the range of possible error types identified. It appears that tasks which involve series of stimuli may be better suited to the assessment of PD deficits than those consisting only of discrete items.

The present study was designed to investigate the nature of impairments associated with PD by manipulating a deductive reasoning task which is sensitive to PD deficits (Channon et al., 1993). In this task, subjects are presented with a series of pairs of four-dimensional stimuli and only one of each pair is correct. They are required to work out which of a set of eight possible rules is in operation by using correct/wrong feedback to narrow down the possible rules. Channon et al. (1993) found that, relative to controls, the PD group achieved fewer correct solutions, tested fewer correct hypotheses, and were less likely to use appropriate lose-shift strategies following negative feedback. However, only the minority of PD lose-stay errors were found to reflect perseveration (selecting the same stimuli already shown to be incorrect by feedback). Typically, their errors involved shifting from one stimulus shown to be incorrect by feedback to another stimulus that previous feedback had already shown to be incorrect. This suggested a failure to attend to or to integrate all the information conveyed by negative feedback, rather than the presence of a perseverative or set shifting deficit. These results suggested that PD deficits may be associated with reduced working memory resources for task performance. It is also possible that PD patients are more vulnerable than controls to the development of inappropriate response sets if they attend to incorrect stimulus dimensions, and receive apparent reinforcement, which later feedback shows to be incorrect. For instance, if the correct solution is white, a stimulus that is both small and white will be designated correct, and an incorrect response set to the dimension of smallness could develop. It is necessary to make use of a later feedback trial that reinforces white, but not small, to arrive at the correct solution.

In order to examine the latter hypothesis, the deductive reasoning task used by Channon et al. (1993) was presented in both serial and parallel form, to determine whether parallel presentation facilitated performance for the PD patients. Parallel presentation of all the relevant stimuli and feedback at the beginning of the problem is known to permit use of a wider range of performance strategies. For instance, subjects can deduce the correct answer to a problem by testing the viability of individual rules one by one in a serial fashion; this is only possible with serial presentation by waiting until the end of the problem, when all the information is available. Alternatively, the implications of one card for all the possible rules can be worked out and combined with the information from additional cards to deduce the answer; this may carry a heavier working memory load than the former strategy. Parallel presentation appears less likely to lead to the development of a strong response set within the problem, because subjects are not channelled into a narrow focus on specific rules which may, later, turn out to be incorrect. Thus, if PD patients are more vulnerable to the development of such response sets, they may show less impairment with parallel presentation.

To examine the hypothesis that a working memory deficit might account for PD impairments, a running memory task was included in the study. This allowed the examination of subjects' capacity to direct attentional resources appropriately in a task that did not require higher-order deductive reasoning. A memory updating task was chosen, in which subjects are given strings of stimuli without knowing their length in advance, and asked to recall a specified number of the most recent items. An appropriate performance strategy involves continually updating the current contents of memory by dropping the earliest items and replacing them with the more recent. Morris and Jones (1990) concluded that this type of task draws on the 
resources of both the $\mathrm{CE}$, which co-ordinates the updating of working memory in real time, and the articulatory loop (AL), which is involved in the serial recall component of the task.

\section{METHODS}

\section{Subjects}

Twenty patients, ten males and ten females, with a diagnosis of idiopathic PD took part in the study. All had an age of onset of 35 or over, and were stabilized on dopaminergic medication (unchanged for a minimum of one month). Twenty control subjects, 11 males and nine females, were selected from a volunteer subject panel. They were matched for age and education. Other selection criteria for both groups included a score of 26 or above on the Folstein Mini Mental State; no diagnosis of major depression or other psychiatric disorder using DSM-III-R criteria; no history of alcohol or drug abuse; and no significant physical illness. The groups did not differ significantly in age (PD 66.0 years, SD 6.6; controls 63.9 years, SD 6.9); Folstein scores (PD 27.6, SD 1.4; controls 28.3, SD 1.5); WAIS$\mathrm{R}$ (Wechsler, 1981) age-scaled vocabulary test (PD 12.3, SD 2.6; controls 12.8, SD 2.0); or years of education (PD 12.2 years, SD 2.8; controls 12.1 years, SD 2.8). The PD group scored significantly higher $(p<0.0001)$ on the Beck Depression Inventory (BDI; Beck et al., 1961) (PD 9.7, SD 3.1; controls 5.7, SD 2.9). Mean duration of illness for the PD group was 6.5 years (SD 2.7) and all patients were prescribed dopaminergic medication. One was taking selegiline alone, two were taking Madopar alone, five Sinemet alone, three Sinemet and selegiline, five Madopar and selegiline, one Madopar and bromocriptine, one Madopar and pergolide, one Madopar, bromocriptine and Sinemet, and one Madopar, selegeline and Sinemet.

After giving informed consent, all subjects carried out the two experimental tasks, in counterbalanced order within each group, and the clinical measures. Full ethical approval was granted for the study.

\section{Deductive reasoning task}

This task was adapted from the deductive reasoning problems described by Levine (1996). A typical procedure is described below (used by Channon et al., 1993). Each problem consisted of a series of trials, with two stimuli on each trial (see Fig. 1). The two stimuli varied on up to four dimensions (large-small, A-B, black-white, left-right), such that one of the two letters in each pair would be large and the other would be small, and so on. A pretraining procedure of one- and two-dimensional problems was used, based on that described by King and Philips (1985). The problems were presented in a selection paradigm, where subjects are presented with predetermined choices and asked to deduce the correct solutions. The correct solution for each problem is thus invariant across subjects. Correct or wrong feedback was presented beside each of the three stimulus cards in each problem, and subjects were asked to work out which of the possible rules could be correct at that stage of the problem (see Fig. 1).

Previous versions of the task have commonly used serial presentation of the four-dimensional problems (see, for example, Channon et al., 1993). The cards are presented individually and, for optimal performance, it is necessary to deduce, for each of the three trials, which of the eight possible rules can still be the correct solution. After the first feedback trial, subjects can eliminate four of the eight rules, retaining four; after the second feedback trial, combination of the knowledge derived from the first and second pieces of feedback makes it possible to eliminate two further rules, thus retaining two rules only. If the second feedback trial was studied in isolation from the first a total of only four rules can be eliminated. On the third feedback trial, all but one of the rules can be eliminated by combining the information from all three trials. Strategies for eliminating incorrect solutions accurately on successive feedback trials are likely to involve a heavy working memory load, since they demand an overview of all eight possible rules. Serial presentation

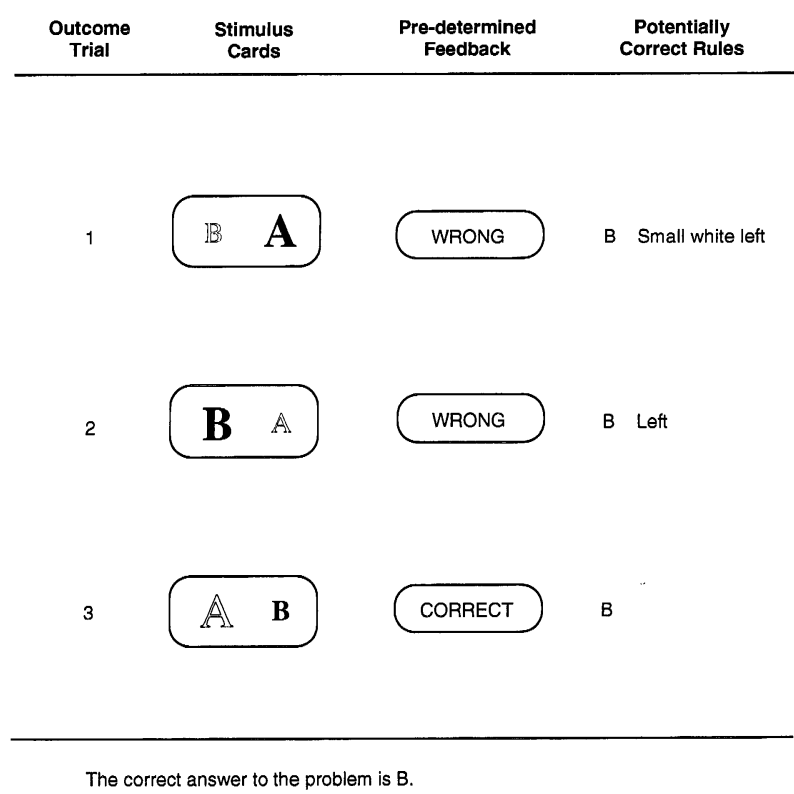

FIG. 1. An example of a four-dimensional problem; the three outcome trials were presented simultaneously in the parallel condition and serially in the serial condition. 
of the information, therefore, constrains the potential strategies which subjects can use in the earlier stages of problem solving. All the information is available to subjects in the final feedback trial only. By contrast, parallel presentation of the same information permits subjects to adopt different strategies, such as testing each possible rule in turn, checking whether it is correct for each of the three feedback trials, and eliminating it as necessary. This procedure can be repeated for each of the eight rules or until the correct solution is found, and it may be less demanding of working memory resources (for example, test black, then white, then left, and so on). Serial and parallel versions were compared in the present study, presented in counterbalanced order.

One- and two-dimensional pretraining problems. The first four problems varied only in one dimension (size, position, letter or colour) and the two stimuli were otherwise identical. Subjects were first introduced to four one-dimensional problems, each varying only in one attribute (size, position, letter or colour) giving two possible rules. For each problem, they were instructed that there were two possible solutions (for example, large or small), and they were shown one card with the word 'correct' or 'wrong' placed next to either the left-hand or the right-hand stimulus. The solutions were selected to represent a different rule each time; the dimension of position (left-right) was inevitably present for each of the problems, but this dimension was introduced as a possible solution for only one of the one-dimensional problems. Subjects were asked to give the solution. If this was incorrect, the same problem was presented again. As the parallel and serial conditions were identical for the one-dimensional problems, only two problems were administered on whichever condition was presented second, and the score consisted of the combined sum of the first two problems for each condition.

The next four problems varied on two dimensions (position and either size, letter or colour), such that each stimulus would be, for example, either black or white and either on the left or on the right. Subjects were instructed that there were four possible solutions and that their task was to discover which one was correct. Two cards were presented, each with the word 'correct' or 'wrong' beside one of the two stimuli on each card. The cards were designed so that the problems could be solved in two feedback trials. After the first feedback trial two of the rules could be eliminated (for example, black and right), and, after the second feedback trial, a further possibility was eliminated (for example, white). In the parallel condition, both cards were presented together and subjects were asked to give the correct overall solution to the problem. In the serial condition, the first card was presented and subjects were asked to state which of the four possible rules could be correct at that stage of the problem. The second card was then presented, leaving the first card on display, and subjects were asked for the correct overall solution to the problem. Again, the solutions to the problems in the parallel and serial conditions were identical and only three problems were presented in whichever condition was presented second; the first three problems were scored for each condition.

Four-dimensional problems. Eight four-dimensional problems were used for each condition. Each varied along all four dimensions simultaneously, giving eight possible rules, and the task was to determine which was the correct one. Subjects were informed of the eight possible rules, and instructed to find the correct solution to each problem. Each problem consisted of three cards, each with the word 'correct' or 'wrong' beside one of the two stimuli on the card. The solutions to the problems differed for each condition. Using three feedback trials, it is possible to arrive at a single correct solution for every possible combination of feedback and response pattern. Feedback as to whether the chosen stimulus is correct or wrong was given according to a predetermined sequence, in order to control exposure to positive and negative feedback. There are eight possible sequences of correct (C) or wrong (W) feedback for each series of three feedback trials (CCC; WWW; CCW; WWC; CWW; WCC; CWC; WCW); following Channon et al. (1993), the latter four sequences were used.

The problems were constructed so that there were always four logically correct rules after the first feedback, two after the second, and one after the third (the correct solution). The procedure was similar to that for the two-dimensional stimuli. In the parallel condition, the three cards were presented together, and subjects were asked to state the correct overall solution to the problem (see Fig. 1). In the serial condition, the three stimuli were presented one at a time, and subjects were asked to state which rules could possibly be correct at each stage of the problem, as well as the final solution. Subjects adopting a perfect strategy could, therefore, narrow the initial set of eight possible rules to four at the first stage, to two at the second stage, and to one final correct solution at the third stage. 


\section{Updating task}

Memory updating tasks require subjects to modify the current contents of working memory continually in order to accommodate new input. This type of paradigm was first described by Pollack et al. (1950), who presented subjects with strings of items of unspecified length and then asked them to perform serial recall on a specific number of the most recent items. Since list length is unknown to the subjects, an appropriate performance strategy involves rehearsal of the items until the required set size is reached. If further items are presented, the set is then updated each time by dropping the oldest item and adding the new one to the end of the list. The present task was based on that described by Morris and Jones (1990). Two conditions were used, requiring recall of series of different lengths (four and six items). Recall of four items is generally below the memory span of most subjects, whereas recall of six items is closer to (or greater than) the memory span of most subjects.

The stimuli were two sets of randomly-ordered series of consonants, each consisting of four practice trials and 16 experimental trials. For one condition, subjects were required to recall verbally the last four letters in the series. This set consisted of four series at each of four lengths; four, six, eight and ten letters. In the other condition, recall involved the last six consonants in the series. This set consisted of four series at each of four lengths; six, eight, ten and 12 letters. Individual trials in both conditions, therefore, required between zero and six updates, depending on the length of the series. Order of presentation of the individual series within each set was randomized. They were presented by audiotape at a rate of one letter per second, and the order of presentation of the two conditions was counterbalanced within each group. Subjects were instructed to listen to each series and, afterwards, to recall the final four or six letters in order of presentation. They were encouraged to give an answer, where possible, for each of the four or six target letters.

The errors made by subjects were classified into three types. Intrusion errors were those in which subjects reported letters which had appeared in the earlier part of the series, before the final four or six letters. Order errors were those in which subjects reported letters which were part of the target four or six letters in the wrong place. Non-series errors were those in which subjects reported letters which had not been in the series or where subjects failed to report a letter at all. All errors were classified into one of these three groups.

\section{RESULTS}

\section{Deductive reasoning task}

Mean scores for the two groups can be seen in Table I. A significance level of 5\% was adopted throughout.

Pretraining problems. A $t$-test was used to examine performance on the one-dimensional training problems, combined for the two conditions since the problems were identical at this level. The difference between the groups approached, but did not reach, significance on this measure $(t(27.94)=1.95$, $p=0.061)$ and both groups approached maximal performance.

For the two-dimensional problems, repeated measures ANOVA with one between-subjects factor (group) and one within-subjects factor (condition: parallel or serial) showed a significant effect of group $(\mathrm{F}(1,38)=9.68, p=0.004)$; the group by condition interaction was not significant $(\mathrm{F}(1,38)=2.43$, $p=0.127)$. The PD patients scored lower than controls for both serial and parallel presentation (See Table I). The main effect of condition was not significant $(\mathrm{F}(1,38)=0.07, p=0.796)$.

Four-dimensional problems. On the four-dimensional problems, there was a significant group difference $(\mathrm{F}(1,38)=13.13, p=0.001)$ in the number of correct solutions achieved and no significant group by condition interaction $(\mathrm{F}(1,38)=0.01, p=0.934)$ or main effect of condition $(\mathrm{F}(1,38)=0.01, p=0.934)$. Again, the PD patients achieved fewer correct solutions than controls (See Table I). For the serial condition, it was possible to analyse the lists of potentially correct rules produced by the subjects after each of the three stages. Omissions and false positive errors

TABLE I: Mean scores and standard deviations for the PD and control subjects on the deductive reasoning problems.

\begin{tabular}{lllll}
\hline & \multicolumn{3}{c}{ PD group } & \multicolumn{2}{c}{ Control group } \\
& Mean & SD & Mean & SD \\
\hline Correct solutions & & & & \\
$\quad$ 1-dimensional & 3.40 & 0.82 & 3.80 & 0.41 \\
2-dimensional parallel & 1.35 & 1.23 & 2.50 & 0.83 \\
2-dimensional serial & 1.60 & 1.19 & 2.15 & 0.93 \\
4-dimensional parallel & 3.25 & 2.49 & 5.75 & 2.40 \\
$\quad$ 4-dimensional serial & 3.25 & 2.27 & 5.80 & 2.42 \\
Errors in 4-dimensional serial & & & & \\
Omissions stage 1 & 1.06 & 1.31 & 0.58 & 0.94 \\
Omissions stage 2 & 0.59 & 0.62 & 0.38 & 0.44 \\
Omissions stage 3 & 0.29 & 0.32 & 0.14 & 0.14 \\
False positives stage 1 & 0.22 & 0.39 & 0.06 & 0.13 \\
False positives stage 2 & 1.32 & 0.81 & 1.01 & 0.82 \\
False positives stage 3 & 1.80 & 1.14 & 1.32 & 1.21 \\
\hline
\end{tabular}


TABLE II: Mean scores for the PD and control subjects on the updating task.

\begin{tabular}{lrrrr}
\hline Correct solutions & \multicolumn{2}{c}{ PD group } & \multicolumn{2}{c}{ Control group } \\
& Mean & SD & Mean & SD \\
\hline Recall-4 Position 1 & 8.35 & 3.18 & 8.80 & 2.21 \\
Recall-4 Position 2 & 9.60 & 3.05 & 11.05 & 1.64 \\
Recall-4 Position 3 & 11.15 & 3.44 & 12.90 & 1.45 \\
Recall-4 Position 4 & 12.85 & 3.90 & 14.90 & 1.02 \\
Intrusion errors & 8.35 & 7.26 & 6.35 & 3.35 \\
Order errors & 9.40 & 9.29 & 6.20 & 2.69 \\
Non-series errors & 4.45 & 4.48 & 3.85 & 2.64 \\
Recall-6 Position 1 & 5.05 & 3.34 & 5.65 & 2.01 \\
Recall-6 Position 2 & 5.35 & 3.23 & 6.15 & 2.30 \\
Recall-6 Position 3 & 4.65 & 3.15 & 5.70 & 2.18 \\
Recall-6 Position 4 & 5.65 & 3.16 & 7.40 & 2.58 \\
Recall-6 Position 5 & 6.90 & 3.52 & 9.40 & 2.56 \\
Recall-6 Position 6 & 9.05 & 3.52 & 12.25 & 2.34 \\
Intrusion errors & 11.85 & 7.73 & 11.90 & 2.99 \\
Order errors & 27.05 & 9.20 & 22.25 & 6.81 \\
Non-series errors & 19.65 & 11.09 & 14.95 & 8.87 \\
\hline
\end{tabular}

were analysed for the three stages of feedback using ANOVA and this analysis showed a significant effect of group $(\mathrm{F}(1,38)=5.94, p=0.02)$; the $\mathrm{PD}$ patients tended to make more of both types of error than controls. There were no significant interactions between group and type of error $(\mathrm{F}(1,38)=0.01, p=0.907)$, group and stage $(\mathrm{F}(1.14), 76)=1.43, p=0.858)$, or group, type and stage $(\mathrm{F}(1.17,76)=2.66, p=0.105)$.

\section{Updating task}

Mean scores for the two groups are shown in Table II. Performance was first examined in both conditions (recall of the last four or six letters) for the trials which required no updating (i.e. series of length four or six respectively). These were equivalent to measures of forward digit span on traditional tests, such as the WAIS-R digit span. Repeated measures ANOVA with one between-subjects factor (group) and one withinsubjects factor (serial position) was carried out separately for the two conditions. The effect of group and the group by serial position interactions were not significant for either the four-length condition $(\mathrm{F}(1,38)=0.62, p=0.437)$ and $(\mathrm{F}(2.78), 114)=0.09$, $p=0.96)$ or the six-length condition $(\mathrm{F}(1,38)=2.74$, $p=0.106)$ and $(\mathrm{F}(5,190)=0.27, p=0.921)$.

Performance was then compared for the two groups on all the trials in each condition. For the four-length recall trials, repeated measures ANOVA was carried out with one between-subjects factor (group) and two within-subjects factors (number of updates: zero, two, four or six serial position: one, two, three or four), to compare the number of letters recalled correctly. The group by serial position effect was significant $(\mathrm{F}(2.31$, $114)=2.97, p=0.049)$. The group effect did not reach significance $(\mathrm{F}(1,38)=3.43, p=0.072)$; nor was there a significant group by updating interaction $(\mathrm{F}(2.84$, $114)=0.87, p=0.456$ ) or group by updating by serial position interaction $(\mathrm{F}(8.82,342)=0.84, p=0.579)$. A comparison of the two groups on the four serial positions, regardless of number of updates, showed that the PD patients tended to make more errors than controls, and that they improved less than controls on later items in the series (see Table II); $t$-tests were not significant, using a strict criterion (alpha $=0.05 / 4$ ).

A similar analysis for the six-length recall condition also showed a significant group by serial position interaction $(\mathrm{F}(3.80,190)=2.58, p=0.042)$ and there was also a significant effect of group $(F(1,38)=5.77$, $p=0.021)$. The group by updating interaction was not significant $(\mathrm{F}(2.83,114)=0.78, p=0.501)$; nor was the group by updating by serial position interaction $(\mathrm{F}(15,570)=1.20, p=0.267)$.

There were highly significant $(p<0.0001)$ main effects of serial position and number of udpates, and position by number of updates interactions, for both the four-length and six-length recall conditions. Mean scores indicated that both groups showed recency effects in that their scores increased for the letters at the end of the series. There was no evidence of primacy effects, since recall of letters at the beginning of the series was poorer than recall of later letters. The need to update the series was associated with poorer recall.

Errors. The rates of intrusion errors, order errors and non-series errors made by the two groups were compared using $t$-tests, and none of the differences between the groups reached significance. Errors involving the order of the target letters were common for both groups in both the four-length and six-length recall conditions. Intrusion errors, in which subjects reported letters from the beginning of the series, occurred at similar rates to order errors in the fourlength recall condition, but they were less common than other error types for both groups in the sixlength recall condition.

Correlations between the experimental measures. Pearson correlation coefficients were calculated between the BDI, duration of illness for the PD group, and total scores for the experimental variables which significantly differentiated the two groups. At the 0.05 level, the BDI did not correlate significantly with any measure for the PD group, but it correlated significantly with total six-length recall scores for the control group $(\mathrm{r}=-0.49, p=0.029)$. Correlations with duration of illness for the PD group did not reach significance for any measure, although they approached significance for total six-length recall 
$(\mathrm{r}=-0.40, p=0.079)$ and false positive errors on four-dimensional serial deductive reasoning $(\mathrm{r}=-0.39, p=0.085)$.

Intercorrelations between the two experimental tasks were also calculated for each group. There were no significant correlations between deductive reasoning and updating for the PD group; for the controls, there were significant correlations between fourdimensional correct solutions and total four-length recall scores $(\mathrm{r}=0.50, p=0.024)$.

\section{DISCUSSION}

The present study was designed to examine the performance of PD patients on two tasks which are sensitive to executive dysfunction, deductive reasoning and memory updating. The findings revealed impairment in the PD group, compared with controls, on both the discrimination learning and memory updating tasks. PD patients gave fewer correct solutions to the reasoning problems for both serial and parallel presentation. The serial presentation permitted examination of omission and false positive error rates in deducing possible rules at each stage of the problems. This showed that PD patients made more errors than controls, both by omitting possible rules and by including rules which feedback had already shown to be incorrect. The finding of impaired reasoning is consistent with a growing body of evidence of deficits in aspects of executive function in medicated PD subjects (see, for example, Downes et al., 1989; Brown and Marsden, 1988; Owen, et al., 1992; Cronin-Golomb et al., 1994) and, to a lesser extent, in unmedicated subjects (see, for example, Downes et al., 1989; Cooper et al., 1991). On the memory updating task, the PD patients reported fewer items correctly than controls but they did not appear particularly prone to errors of intrusion from earlier items in the list. The PD group had slightly higher BDI scores than the control group, but this probably reflected symptoms of physical PD pathology, and it did not correlate with performance on the experimental variables which differentiated the groups; duration of illness. Nor did it correlate significantly with the experimental variables which differentiated the groups, although there was a tendency for longer duration of illness to be associated with poorer scores on one of the measures for each task.

It was hypothesized that, if PD impairment is attributable to a tendency to develop a strong response set, with subsequent set shifting deficits, then PD patients may show greater impairment on the serial deductive reasoning problems than on the parallel deductive reasoning problems, because the former category may encourage the development of a response set. This hypothesis was not supported by the main finding, that PD patients were impaired in giving correct solutions to both serial and parallel problems, at both two- and four-dimensional levels of difficulty.

Since there were no significant interactions with condition or main effect of condition (serial or parallel presentation), there was no evidence to show that either group used different strategies to arrive at solutions in the two conditions. There are at least two possible explanations for this. It may be that neither group was using an efficient strategy in either of the two conditions and, thus, did not benefit from the opportunity to use a different approach in the parallel condition. In favour of this is the finding that, although the PD subjects performed more poorly than controls, neither group approached maximal performance for the fourdimensional problems, suggesting that the performance strategies used by both groups were not optimal. Alternatively, it may be that both groups tended to base their solutions to the problems on the information available at the end of the serial problems, at which stage all the information needed to solve the problems was displayed simultaneously. By this stage, the two modes of presentation had become equivalent, and, since time limits were not used, this was an appropriate strategy for achieving the final solution.

Regardless of the explanation for the lack of difference in performance on the two versions of the task, the most important finding is that PD patients were impaired to a similar extent under parallel presentation conditions and serial presentation conditions. This makes it difficult to sustain an explanation of their deficits in the the present task in terms of a strong tendency to form response sets to previously reinforced items, and subsequent difficulties in shifting away from this, as concluded by Cronin-Golumb et al. (1994) from their studies in PD patients. Moreover, PD error patterns, including the proportion of intrusion errors, did not differ significantly from controls on the memory updating task. PD patients were no more susceptible to difficulties in suppressing previously presented items on series which required memory updating. Alternatively, it was hypothesized that PD impairment on executive tasks might arise from reduced working memory resources available for task performance. Such resources are necessary in the deductive reasoning task employed in this study to remember the possible correct rules and integrate the feedback. Evidence in favour of this hypothesis is the finding that the PD group was impaired not only in deductive reasoning, but also in the memory updating task, which does not involve deductive reasoning. Other evidence is accumulating which suggests that cognitive impairment in PD may be at least partially attributable to reduced 
resources affecting working memory (see, for example, Cooper and Sagar, 1993; Cooper et al., 1993). Theories which relate to working memory deficits which have been offered in explanation of executive impairment in PD include instability of response set as a result of difficulties in attending to previously unreinforced stimuli (Downes et al., 1989), and impaired internal versus external control of attention due to reduced SAS resources (Brown and Marsden, 1988). The precise nature of the observed deficits is likely to vary with the particular task characteristics, and reduced working memory resources at the level of the CE (or SAS) could, presumably, underlie a variety of patterns of impaired performance on different executive tasks relating to difficulties in initiating or sustaining appropriate strategies.

In summary, PD patients in the present study showed evidence of impairment both on a deductive reasoning task and on a working memory task. The concept of impaired set shifting does not appear to provide an adequate characterization of the nature of the observed PD deficits, and an increased focus of attention on the availability and efficiency of working memory resources may be more fruitful.

\section{Acknowledgements}

I am grateful to Dr. G. Stern, Dr. A.J. Lees and Susan Bishop for clinical co-operation and to Robert Sanders and Maire Sharkey for assistance with part of the data collection.

\section{REFERENCES}

Alexander GE, DeLong MR and Strick PL (1986) Parallel organisation of functionally segregated circuits linking basal ganglia and cortex. Annual Review of Neuroscience 9, 357-381.

Baddeley AI and Hitch GJ (1974) Working memory. In: Recent advances in learning and motivation (Ed G Bower) Vol 3. Academic Press, New York, pp. 47-90.

Beck AT, Ward $\mathrm{CH}$, Mendelson M, Mock J and Erbaugh J (1961) An inventory for measuring depression. Archives of General Psychiatry 4, 561-571.

Bowen FP, Kamienny RS, Burns MM and Yahr MD (1975) Parkinsonism: Effects of levodopa treatment on concept formation. Neurology 25, 701-704.

Brown RG and Marsden CD (1988) Internal versus external cues and the control of attention in Parkinson's disease. Brain 111, 323-345.

Channon S, Jones MC and Stephenson S (1993) Cognitive strategies and hypothesis testing during discrimination learning in Parkinson's disease. Neuropsychologia 31, $75-82$.

Cools AR, van den Bercken JHL, Horstink MWI, van Spaendonck KPM and Berger HJC (1984) Cognitive and motor shifting aptitude disorder in Parkinson's disease. Journal of Neurology, Neurosurgery and Psychiatry 47, 443-453.

Cooper JA and Sagar HJ (1993) Incidental and intentional recall in Parkinson's disease: An account based on dimin- ished attentional resources. Journal of Clinical and Experimental Neuropsychology 15, 713-731.

Cooper JA, Sagar HJ, Jordan N, Harvey NS and Sullivan EV (1991) Cognitive impairment in early, untreated Parkinson's disease and its relationship to motor disability. Brain 114, 2095-2122.

Cooper JA, Sagar HJ and Sullivan EV (1993) Short-term memory and temporal ordering in early Parkinson's disease: Effects of disease chronicity and medication. Neuropsychologia 31, 933-949.

Cronin-Golomb A, Corkin S and Growdon JH (1994) Impaired problem-solving in Parkinson's disease: Impact of a set-shifting deficit. Neuropsychologia 32, 579-593.

Downes IJ, Roberts AC, Sahakian BJ, Evenden JL, Morris RG and Robbins TW (1989) Impaired extra-dimensional shift performance in medicated and unmedicated Parkinson's disease: Evidence for a specific attention dysfunction. Neuropsychologia 27, 1329-1342.

Flowers KA and Robertson C (1985) The effects of Parkinson's disease on the ability to maintain a mental set. Journal of Neurology, Neurosurgery and Psychiatry 48, 517-529.

Gotham AM, Brown RG and Marsden CD (1988) "Frontal" cognitive functions in patients with Parkinson's disease "on" and "off" levodopa. Brain 111, 299-321.

King S and Philips S (1985) Problem-solving characteristics of process and reactive schizophrenics and affectivedisordered patients. Journal of Abnormal Psychology 94, 17-29.

Lees AJ and Smith E (1983) Cognitive deficits in the early stages of Parkinson's disease. Brain 106, 257-270.

Levine M (1966) Hypothesis behavior by humans during discrimination learning. Journal of Experimental Psychology 71, 331-338.

Milner B (1964) Some effects of frontal lobectomy in man. In: The Frontal Granular Cortex and Behaviour (Eds JM Warren and K Akert). McGraw Hill, New York, pp. 313-334.

Morris N and Jones DM (1990) Memory updating in working memory: The role of the central executive. British Journal of Psychology 81, 111-121.

Norman DA and Shallice T (1986) Attention to action: Willed and automatic control of behavior. In: Consciousness and Self-Regulation: Advances in Research and Theory, Vol 4 (Eds RJ Davidson, GE Schwartz and D Shapiro). Plenum, New York.

Owen AM, James M, Leigh PN, Summers BA, Marsden CD, Quinn NP, Lange KW and Robbins TW (1992) Fronto-striatal cognitive deficits at different stages of Parkinson's disease. Brain 115, 1727-1751.

Pillon B, Dubois B, Lhermitte F and Agid Y (1986) Heterogeneity of cognitive impairment in progressive supranuclear palsy, Parkinson's disease, and Alzheimer's disease. Neurology 36, 1179-1185.

Pollack I, Johnson L and Knaft P (1959) Running memory span. Journal of Experimental Psychology 57, 137-146.

Sandson J and Albert ML (1987) Perseveration in behavioral neurology. Neurology 37, 1736-1741.

Taylor AE, Saint-Cyr JA and Lang AE (1986) Frontal lobe dysfunction in Parkinson's disease. Brain 109, 845-883.

Wechsler D (1981) Adult Intelligence Scale - Revised Manual. Psychological Corporation, New York.

(Received 31 January 1996; accepted 21 March 1997) 


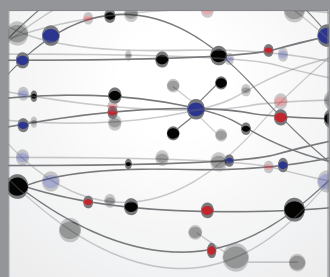

The Scientific World Journal
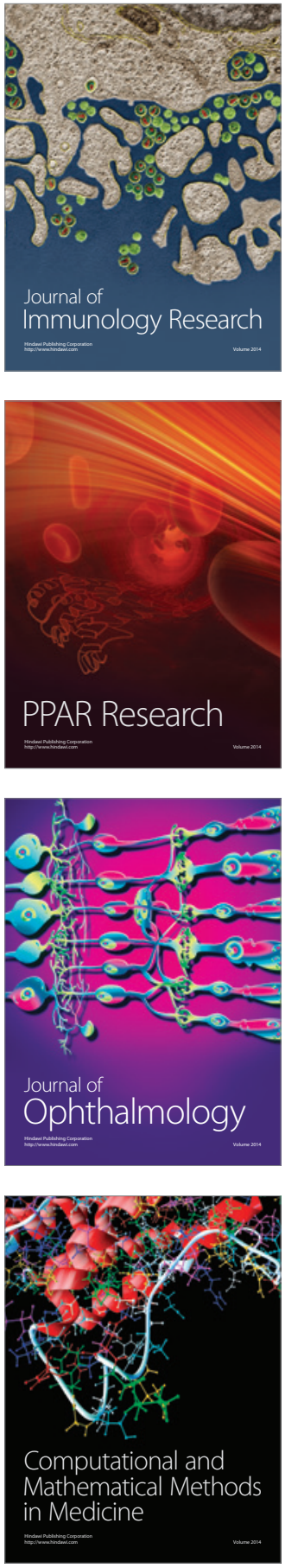

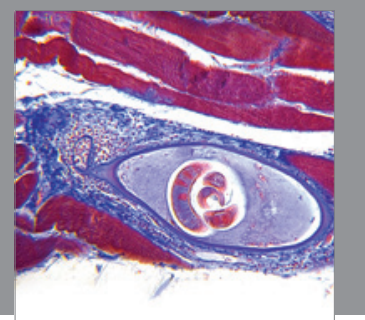

Gastroenterology

Research and Practice
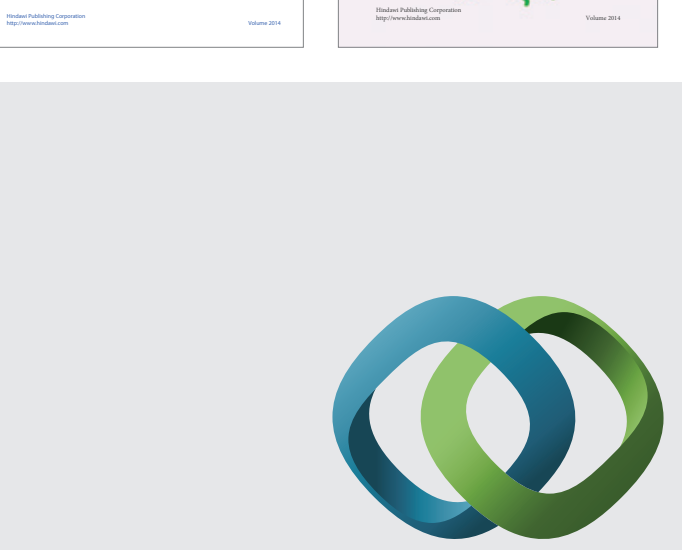

\section{Hindawi}

Submit your manuscripts at

http://www.hindawi.com
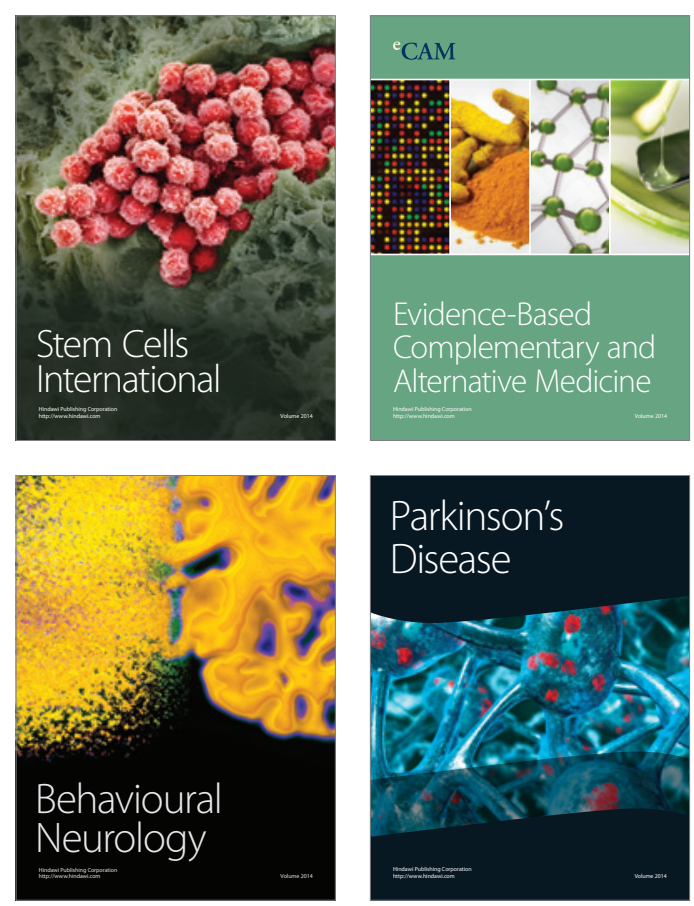

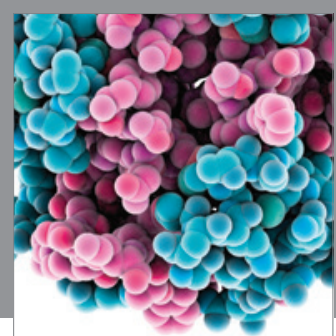

Journal of
Diabetes Research

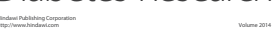

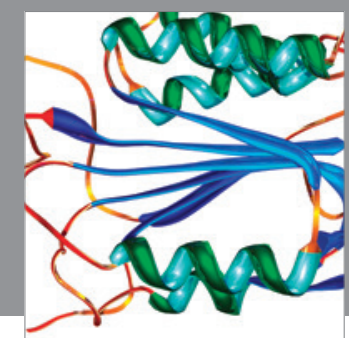

Disease Markers
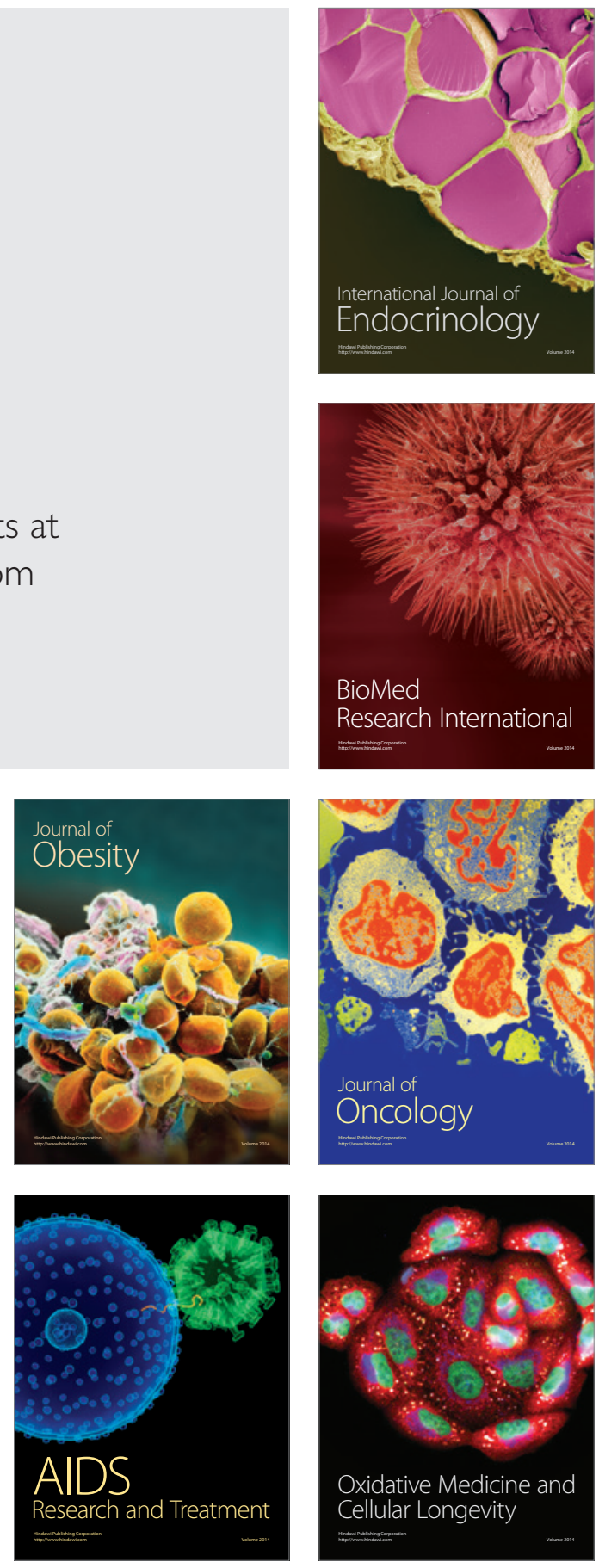